cross over the wing margin and, whether they are dorsal or ventral, meet at a common boundary ${ }^{14}$. At the $\mathrm{A} / \mathrm{P}$ border it is believed that cells do not mix because they have different surface properties; there is thought to be a 'label' on the posterior cells due to the action of the engrailed selector gene ${ }^{8,9}$. But - for both the $\mathrm{D} / \mathrm{V}$ and thorax/wing compartments - no selector genes were known.

However, in the mid-1980s a zone of cells that divide rather little was discovered near the $\mathrm{D} / \mathrm{V}$ but not the $\mathrm{A} / \mathrm{P}$ boundary, and it was suggested that these cells could act as a barrier to the growth of clones across the $\mathrm{D} / \mathrm{V}$ wing margin $^{15}$. If this were the mechanism it would mean that $\mathrm{D} / \mathrm{V}$ and $\mathrm{A} / \mathrm{P}$ compartments are made differently. Following this, an influential review ${ }^{16}$ discussed the idea that the wing disk is sequentially divided up into true compartments, and then ceremoniously buried it.

Blair $^{1}$ and Diaz-Benjumea and Cohen ${ }^{2}$ have opened up this particular grave. They show, with beautiful evidence, that the wing disk is divided into dorsal and ventral compartments by a selector gene. The gene is apterous, which encodes a transcription factor containing both a homeodomain and a LIM domain ${ }^{17}$. In apterous ${ }^{-}$flies, wings and halteres (homologous to the hind wings of other insects) fail to develop, but the thoracic trunk is almost normal; the remaining parts of the fly are unaffected. The apterous protein is found in the dorsal region of the wing disk from early second instar when the disk contains fewer than 200 cells $^{18}$. This is at the time when the $\mathrm{D} / \mathrm{V}$ boundary comes into existence, as defined by cell lineage $\mathrm{e}^{12}$. Both the borders of marked clones and the limit of apterous expression coincide exactly for many cells.

Blair demonstrates that this boundary runs along the middle of the zone of 'non-proliferating' cells previously proposed to act as a barrier; the dorsal clones (which express apterous) include one half of the zone, and the ventral the other results that comprehensively disprove the barrier hypothesis. Furthermore, the dorsal expression of apterous is found in both anterior and posterior compartments, even though these polyclones were separated in the young embryo and belong to different parasegments. This illustrates the way in which selector genes combine to specify development of compartments.

Diaz-Benjumea and Cohen also made clones of cells that lack the apterous gene. In the ventral compartment, the clones develop normally - hardly surprising, because the gene is not expressed there. But in the dorsal compartment, the apterous $^{-}$clones make apparently perfect ventral cells If they are initiated when the disk contains only tens of cells, they usually join their ventral colleagues and become subsumed into the ventral com- partment when it is formed. If they are made later and away from the $\mathrm{D} / \mathrm{V}$ border, they grow as patches of ventral cells in a dorsal sea; new D/V boundaries, with characteristic bristles, form around their perimeters.

The creation of a new and circular border has a fascinating consequence: it induces a localized outgrowth of the wing with a normal proximodistal pattern. An organizing role for the border in growth had been noted before ${ }^{9}$ but, as DiazBenjumea and Cohen explain, this new observation helps explain why the wing itself grows out. It seems likely that, in normal development, the creation of dorsal cells, which is associated with the activation of apterous in the second larval stage, is in itself enough to trigger the formation of a compartment border, with all its special properties. The border probably represents the upper (or lower) limit of a gradient field. If such a limit (say 10) is formed in a part of the field at a different value (say 7) there will be a blending interaction between border and surround, leading to closely packed intermediate values $(8,9)$ and therefore rapid growth ${ }^{19}$.

Finally, the two new papers should invigorate the search for compartments elsewhere in the fly and, more importantly, in other animals. In our opinion the compartment hypothesis has not been sufficiently applied outside insects, for example to the development of vertebrates. Even so, the rhombomeres in the vertebrate hind-brain are being imaginatively investigated and the evidence that they are compartments - both as units of cell lineage $e^{20}$ and selector gene action ${ }^{21}$ is accumulating nicely.

Peter A. Lawrence is in the MRC Laboratory of Molecular Biology, Hills Road, Cambridge $C B 22 Q H$, UK. Ginés Morata is at the Centro de Biologia Molecular, Universidad Autonoma de Madrid, Madrid 28049, Spain.

1. Blair. S. S. Development 119. 339-351 (1993),

. Diaz-Benjumea. F. \& Cohen, S. Cell 75, 741-752 1993

Lawrence, P. A. Nature New Biol. 242, 251-253 (1973)

. Garcia-Bellido. A. Ripoll, P. \& Morata. G. Nature New Biol. 245. 251-253 (1973).

5. Garcia-Bellido, A. CIBA Fon. Symp. 29. 161-182 (1975)

6. Garcia-Bellido, A., Lawrence, P. A. \& Morata, G. Scient Am. 241, 102-110 (1979)

Struhl. G. Proc natn. Acad. Sci. U.S.A. 79. 7380 (1982)

8. Morata, G. \& Lawrence, P. A. Nature 255, 614,617 (1975)

Lawrence. P. A. \& Morata, G. Devi Biol. 60, 321-337 (1976)

10. Steiner. E. Roux Archiv. dev/ Biol. 180. 9-30 (1976)

11. Struhl. G. Nature 270. 723-725 (1977)

12. Morata, G. \& Lawrence, P. A. Devi Biol. 70, 355 (1979)

13. Kornberg. T. Devi Biol. 86. 363-372(1981)

14. Bryant, P J. Dev/Biol 22 389-411(1970)

15. O'Brochta, D. A. \& Bryant. P. J. Nature 313, 138 (1985)

6. Brower D

17. Cohen. B.. McGuffin. M. E., Pfeiffe, C., Segal, D. \& Cohen, S. M. Genes Dev. 6. 715-729(1992).

18. Williams, J. A., Paddock, S. W. \& Carroll, S. B. Development 117, 571-584 (1993)

19. Lawrence, P. A. The Making of a Fly (Blackwell Scientific, Oxford, 1992)

20. Guthrie. S. et al. Nature 356, 157-159 (1992).

21. Guthrie. S., Prince. V. \& Lumsden, A. Development 118 527-538(1993).

\section{Solar wind}

A NEW defence against Earth-impacting asteroids has recently been proposed by H. J. Melosh and I. V. Nemchinov (Nature $366,21 ; 1993)$. A huge solar mirror, flying alongside the asteroid, could focus sunlight to a point on its surface. The resulting steady plume of evaporation would slowly perturb the asteroid's orbit away from fatal impact with the Earth.

Daedalus is taking this idea a step further. Suppose, he says, that the exact sub-solar point of the asteroid could be suddenly heated into such a hotspot. The resulting plume of vapour would form a refractive volume in space, a sort of gas-lens with a cylindrical axis of symmetry pointing towards the Sun. It would tend to bend sunlight inwards towards the hotspot, mirage-fashion. Lenses like this, which focus by means of refractive-index gradients, are well known to biologists. Our own eyes use this strategy; and certain fishes have eyes whose rod-like lenses focus almost entirely by their cylindrical refractive symmetry. If the plume of expanding gas from the asteroidal hotspot happened to focus the sunlight exactly onto its source, it would be self-maintaining.

DREADCO's physicists are working out the details. What temperature, shape and depth makes a hotspot self-maintaining? How fast can it move over the surface of a spinning asteroid, always tracking the sub-solar point? If a small perturbation defocuses the sunlight, under what conditions will the plume react to refocus it? From the answers, Daedalus hopes to devise the ultimate anti-asteroid strategy. The celestial menace will be attacked, not with a nuclear bomb or a vast solar mirror, but with a precise charge of smokeless powder fired exactly at its sub-solar point. The resulting plume of expanding gas will focus sunlight onto its source, and keep the evaporation going. Under the steady thrust of its new solar. powered rocket motor, the asteroid will slowly diverge away into a non. threatening orbit.

This elegant scheme also lends itself to propelling spacecraft, at least away from the Sun. A small craft made of a volatile material like ammonium chloride might capture enough solar energy to accelerate quite rapidly. But as a terrestrial defence, the idea should be tried out first on a harmless asteroid. As the white-hot crater or equatorial channel of rotation dug its way into the object, its changing brightness and emission spectrum could identify the material being evaporated and monitor its motor action. It would be rank bad luck if the propulsive effect of the hotspot set the asteroid on a collision course with the Earth. David Jones 